\title{
Efficient Infinite-swept Wing Solver for Steady and Unsteady Compressible Flows
}

\author{
Matteo Franciolini ${ }^{\mathrm{a}}$, Andrea Da Ronch ${ }^{\mathrm{b}, *}$, Jernej Drofelnik $^{\mathrm{b}}$, Daniella Raveh ${ }^{\mathrm{c}}$, Andrea \\ Crivellini ${ }^{\mathrm{a}}$ \\ ${ }^{a}$ Department of Industrial Engineering and Mathematical Science \\ Università Politecnica delle Marche, Ancona 60131, Italy \\ ${ }^{b}$ Faculty of Engineering and the Environment \\ University of Southampton, Southampton SO17 1BJ, U.K. \\ ${ }^{c}$ Faculty of Aerospace Engineering \\ Technion, Haifa 32000, Israel
}

\begin{abstract}
An efficient Navier-Stokes solver for the infinite-swept wing problem is presented. The new flow solution, that reproduces correctly the physics responsible for cross-flow effects, is obtained around a two-dimensional stencil. On the contrary, existing state-of-the-art methods rely on a three-dimensional stencil. Numerical details are followed by an extensive validation campaign, including steady and unsteady compressible flows. The test cases are for single and multielement aerofoils in both laminar and turbulent regimes. Under identical conditions (numerical settings, grids, etc.), the computational cost of the proposed solver was reduced by at least $75 \%$ compared to that of existing state-of-the-art methods. This was also confirmed employing various turbulence models. With a limited effort required to enhance an existing computational fluid dynamics solver (either two or three-dimensional), the infinite-swept wing method was implemented in an industrial-grade package used across Europe for rapid engineering analysis.
\end{abstract}

Keywords: rapid CFD methods, laminar flows, turbulent flows, steady and unsteady problems, multi-element aerofoil

\section{Nomenclature}

$\begin{array}{ll}b & \text { Wing span, [m] } \\ k & \text { Reduced frequency } \\ M & \text { Mach number } \\ \mathbf{R} & \text { Rotation matrix } \\ u, v, w & \text { Velocity components, [m/s] } \\ \mathbf{V} & \text { Velocity vector, [m/s] } \\ \operatorname{Re} & \text { Reynolds number } \\ \operatorname{Pr} & \text { Prandtl number }\end{array}$

${ }^{*}$ Corresponding author. AIAA Senior Member.

Email address: A.Da-Ronch@soton.ac.uk (Andrea Da Ronch) 
II

S

$\dot{\mathbf{q}}$

2 Greek

$\alpha$

$\beta$

$\Lambda$

3 Operators

:

$\otimes$

4 Abbreviations

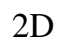

3D

AR

CFD

ISW

LLT

MG

NS

NLF

RANS

SA

URANS

VLM

5 Indexes

'

0

A

$\infty$

:

$\otimes$
Stress tensor

Velocity gradients tensor

Heat flux vector

Angle of attack, [deg]

Side-slip angle, [deg]

Wing sweep angle, [deg]

double scalar product

dyadic product

Two-dimensional

Three-dimensional

Aspect ratio

Computational fluid dynamics

Infinite swept wing

Lifting line theory

Maturity gate

Navier-Stokes

Natural Laminar Flow

Reynolds-averaged Navier-Stokes

Spalart-Allmaras

Unsteady Reynolds-averaged Navier-Stokes

Vortex lattice method

Body-attached frame of reference

Mean value

Amplitude

Freestream

double scalar product

dyadic product

\section{1. Introduction}

Industrial aircraft design proceeds through a series of maturity gates (MG). At the early stages

8 of this process, designers explore a large parameter space relying heavily on empirical and linear 
correlations [1]. In order to down-select the final aircraft concept, the design parameters are tightened and addressed in ever increasing detail [2]. At MG 5, denoted "freeze of concept" in industrial jargon, the shape and structural layout are converged and the aircraft target loads are set. Design target loads are the limiting loads that an aircraft or aircraft component must be designed to withstand. The objective of MG 5 is to anticipate the certification loads level, and issue this data as target loads. It is critical to limit the risk in setting these target loads [3] because: a) if the target loads are underestimated, as revealed following flight test, then expensive re-design is often required incurring the costs and penalties arising from programme delay; and b) if the target loads are overestimated, the aircraft will be heavier than needed with degraded performances.

The analysis process to establish limit loads is computationally demanding as it consists of a very large number of conditions across the loads envelope. Despite a number of simplifications are introduced (linearized aerodynamics, weak coupling between disciplines, etc.), the number of load cases for certification [4], including ranges in Mach number, altitude, payload and fuel mass, exceeds easily several hundreds of thousands.

Today, the solution of the Navier-Stokes (NS) equations is recognized as a prerequisite for realistic flow applications, but the associated computational costs of the three-dimensional (3D) problem become prohibitive when confronted with the number of load cases. Therefore, researchers have proposed two stratagems to overcome this problem. The first stratagem concerns the approximation of the output quantities of interest, e.g. aerodynamic loads, across the design envelope exploiting efficient and accurate adaptive design of experiments [5] and surrogate modelling techniques [6]. The advantage is that the use of off-the-shelf computational fluid dynamics (CFD) packages is straightforward. The second stratagem consists of applying a number of simplifying assumptions in the solution of the NS equations, making calculations cheaper [7, 8]. The advantage of this approach is the ability to find, for a particular problem, a balance between the approximation of the solution and the computational efficiency of the approximation. This work, specifically, addresses the second point.

Rapid CFD methods currently employed in pre-MG 5 are derived combining Prandtl's lifting line theory (LLT) or the vortex lattice method (VLM), which are linear 3D aerodynamic methods, with a two-dimensional (2D) solution of the NS equations. The resulting aerodynamic predictive tool, often referred to as the quasi-3D method, is nonlinear because sectional flow nonlinearities are obtained from a 2D CFD analysis. As the LLT or the VLM are inexpensive, the overall cost of a quasi-3D analysis is comparable to that of a 2D CFD analysis. Reference [9] discussed the design process of the high-lift devices of an Airbus A380-like configuration and the relative challenges encountered in the development phase. The aerodynamic design was built around the quasi-3D method from the early stages of the design process to obtain a pre-optimised shape that was wind tunnel tested. Reference [10] exercised the quasi-3D method for the optimisation of a flexible high-lift wing configuration. Another application concerning drag minimization was presented in Ref. [11]. Therein, the VLM was corrected with the MSES aerofoil predictions [12] based upon the solution of the Euler equations coupled with an integral formulation of the boundary layer equations, with a built-in transition model. The resulting tool was limited to low Reynolds number aerofoils. Other application areas of the quasi-3D method may be found in Refs. [2, 13].

The reasons that the quasi-3D aerodynamic method finds large applicability for industrial design are: a) no detailed 3D geometry information is needed, relying instead on planform data and known aerofoil sections from available databases; b) minimum computational requirements, often not more than several hours of wall clock time for complete polars at various Mach num- 
bers; and c) the easiness to introduce multi-physics considerations (icing, control sizing and allocation, etc.) without extra complication. It is worth observing that the references mentioned in the previous paragraph, and the references therein, rely on a $2 \mathrm{D}$ flow analysis to correct the predictions obtained from a linear 3D aerodynamic model. This is a poor choice in lieu of various experiments [14] showing that cross-flow effects, around a swept wing, strongly influence the boundary layer separation as well as the position of shock waves. Generally, these un-modelled effects are included via knowledge-based corrections, which are also a source of inaccuracies for less-conventional wing planforms departing from the original database.

This study is part of a larger on-going effort at the University of Southampton to deliver, within an industrial design environment, novel computationally efficient methods to calculate dynamic aeroelastic loads around complete aircraft. The aim of this work is to report on the development of a computationally efficient aerodynamic method suitable for aircraft preliminary sizing studies, improving upon existing state-of-the-art methods. The technical objectives are to: a) discuss the resolution of the NS equations for the specific problem of an infinite-swept wing (ISW) on a 2D grid stencil; b) present a thorough validation study of the proposed method for a number of steady and unsteady flow problems, using two different turbulence models; and c) demonstrate and quantify the performance gains for industry-relevant test cases. The proposed methodology has been implemented within the DLR-Tau flow solver, where it is referred to as the $2.5 \mathrm{D}+$ approach to recall the enhanced (computational and convergence) properties in comparison with existing methods. To note that our work goes beyond that presented in Ref. [15] where steady-state flows are considered around simple configurations and an assessment of the performance improvements is missing.

Direct applications of the $2.5 \mathrm{D}+$ solver within an industrial setting are the exploration of the flight envelope for a fixed configuration, including transient analyses when needed, and the optimisation of the aerodynamic shape (control effectors size and allocation, wing twist, etc.).

The paper continues in Section 2] with a brief overview of the CFD solver used in this work. Section 3 explains the underlying methodology and discusses the implementation details of the proposed flow analysis. Then, Section 4 focuses on results for steady and unsteady flow problems. Finally, conclusions are given in Section 5

\section{Flow Solver}

The flow solver employed in this study is DLR-Tau [16], a finite volume based CFD flow solver used by a number of aerospace industries across Europe. The DLR-Tau solver uses an edge-based vertex-centred scheme, where the convective terms are computed via several firstand second-order schemes, including central and upwind types. The viscous terms are computed with a second-order central scheme. Time integration is performed either with various explicit Runge-Kutta schemes or the Lower-Upper Symmetric Gauss-Seidel (LU-SGS) implicit approximate factorization scheme. For time accurate computations, the dual time stepping approach of Jameson [17] is employed. Convergence rate is improved with a multi-grid acceleration technique based on agglomerated coarse grids generated by a pre-processing tool. Several models for turbulence closure are available including, for example, the one-equation SpalartAllmaras (SA) type and more complex two-equation models of the $\mathrm{k}-\omega$ family.

The proposed 2.5D+ method has been implemented within the DLR-Tau flow solver, and is now available from version 2016.1.0 for production [18]. 


\section{Infinite Swept Wing Model}

The ISW model assumes a wing of infinite span with a moderate wing sweep angle, $\Lambda$. In analogy with Euler-Bernoulli beam formulation, the only hypothesis is that the cross section is constant or slowly varying along the wing span. The solution of the ISW model is valid at a reasonable distance from the fuselage and wing tip. Un-modelled effects may be accounted for using the LLT or VLM, as already discussed in Section 1 .

A schematic of an ISW is illustrated in Fig. 1 Two convenient frames of reference (FoRs) are shown. The global FoR with axes $(x, y, z)$, often located at the nose of the aircraft configuration, is generally used to solve the governing flow equations. Then, a body-attached FoR with axes $\left(x^{\prime}, y^{\prime}, z^{\prime}\right)$ provides a suitable choice for the ISW model. The FoR is located at the leading-edge of the wing. The $x^{\prime}$ axis is perpendicular to the quarter-chord axis of the local chord, pointing at the trailing-edge, and the $y^{\prime}$ axis is parallel to the quarter-chord axis of the local chord, pointing at the wing tip.

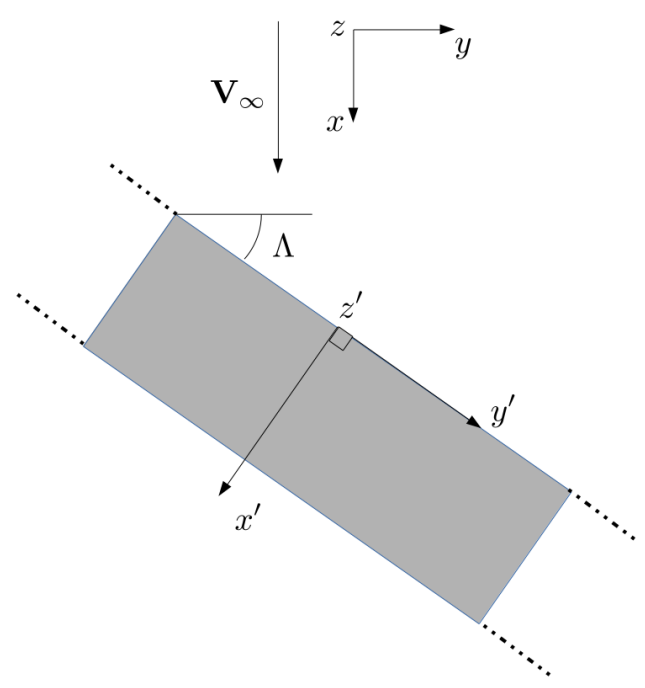

Figure 1: Schematic of an infinite swept wing; flow from top to bottom

The existing state-of-the-art approach to model the ISW involves a one-cell width 3D stencil and can be implemented in two ways, see Fig. 2, with identical results and equivalent computational costs. The first, denoted "sheared approach", solves the 3D NS equations on a wing section extracted along the direction of the incoming flow, i.e. $x-z$ plane in Fig. 11. Boundary conditions include periodicity on the two planes aligned with the flow direction. The freestream angle of attack, $\alpha_{\infty}$, and speed, $U_{\infty}$, are set at the far-field. It is worth noting that in this approach the geometry already incorporates the wing sweep angle. The second approach, denoted "beta approach", solves the 3D NS equations on a wing section extracted along the $x^{\prime}-z^{\prime}$ plane in Fig. 1 Periodic boundary conditions are set on the two planes perpendicular to the $y^{\prime}$ axis. As opposed to the first strategy, any information related to the sweep angle is introduced as an appropriate 
boundary condition at the far-field. The freestream velocity vector, as seen in the global FoR,

$$
\mathbf{V}_{\infty}=\left(\begin{array}{c}
V_{\infty} \cos \left(\alpha_{\infty}\right) \\
0 \\
V_{\infty} \sin \left(\alpha_{\infty}\right)
\end{array}\right)
$$

123

is used to calculate the components of the velocity vector in the body-attached FoR

$$
\mathbf{V}_{\infty}^{\prime}=\mathbf{R} \mathbf{V}_{\infty}=\left(\begin{array}{c}
V_{\infty} \cos \left(\alpha_{\infty}\right) \cos (\Lambda) \\
V_{\infty} \cos \left(\alpha_{\infty}\right) \sin (\Lambda) \\
V_{\infty} \sin \left(\alpha_{\infty}\right)
\end{array}\right)
$$

where $\mathbf{R}$ is the rotation matrix obtained by a rotation $\Lambda$ around the $z$ axis. One then finds that the angle of attack in the body-attached FoR is computed as

$$
\tan \left(\alpha_{\infty}^{\prime}\right)=\frac{w^{\prime}}{u^{\prime}}=\frac{V_{\infty} \sin \left(\alpha_{\infty}\right)}{V_{\infty} \cos \left(\alpha_{\infty}\right) \cos (\Lambda)}=\frac{\tan \left(\alpha_{\infty}\right)}{\cos (\Lambda)}
$$

The side-slip angle in the body-attached FoR, $\beta_{\infty}^{\prime}$, is obtained as

$$
\begin{aligned}
\sin \left(\beta_{\infty}^{\prime}\right) & =\frac{v^{\prime}}{\sqrt{u^{\prime 2}+v^{\prime 2}+w^{\prime 2}}} \\
& =\frac{V_{\infty} \cos \left(\alpha_{\infty}\right) \sin (\Lambda)}{\sqrt{V_{\infty}^{2} \cos ^{2}\left(\alpha_{\infty}\right) \cos ^{2}(\Lambda)+V_{\infty}^{2} \cos ^{2}\left(\alpha_{\infty}\right) \sin ^{2}(\Lambda)+V_{\infty}^{2} \sin ^{2}\left(\alpha_{\infty}\right)}} \\
& =\cos \left(\alpha_{\infty}\right) \sin (\Lambda)
\end{aligned}
$$

For low angles of attack, the approximation $\beta_{\infty}^{\prime} \approx \Lambda$ holds. Equations (3) and (4) represent the far-field boundary conditions for the second ("beta") approach.

The two approaches above solve the NS equations on a one-cell width 3D stencil with periodic boundary conditions set on both planes. For this reason, the convention to denote the "sheared" and "beta" approaches as "3D-stencil" is used hereafter, see Fig. 2. The following Sections illustrate the derivation, implementation, and application of a novel strategy for ISW calculations, also reported in Fig. 2, with a computational cost equivalent to a 2D analysis. The proposed model is referred to as $2.5 \mathrm{D}+$ and uses a purely $2 \mathrm{D}$ stencil.

\subsection{Specific form of the Navier-Stokes Equations}

To solve the NS equations for the ISW model in a very efficient manner, one starts from the 3D NS equations that are generally expressed in the global FoR. The key idea is to avoid imposing the ISW flow through a periodicity boundary condition, using a 3D stencil, but rather a-priori imposing the $y^{\prime}$-independence within the equations. For this reason, the NS equations are rewritten in the body-attached FoR, with axes $\left(x^{\prime}, y^{\prime}, z^{\prime}\right)$. In this FoR, the flow is assumed to have statistical homogeneity in the $y^{\prime}$ direction. This assumption encapsulates a fully developed local flow in the $y^{\prime}$ direction, leading to $\partial(\cdot) / \partial y^{\prime}=0$. The governing equations are therefore simplified by the condition $\partial(\cdot) / \partial y^{\prime}=0$. Once these steps are carried out, the explicit set of 


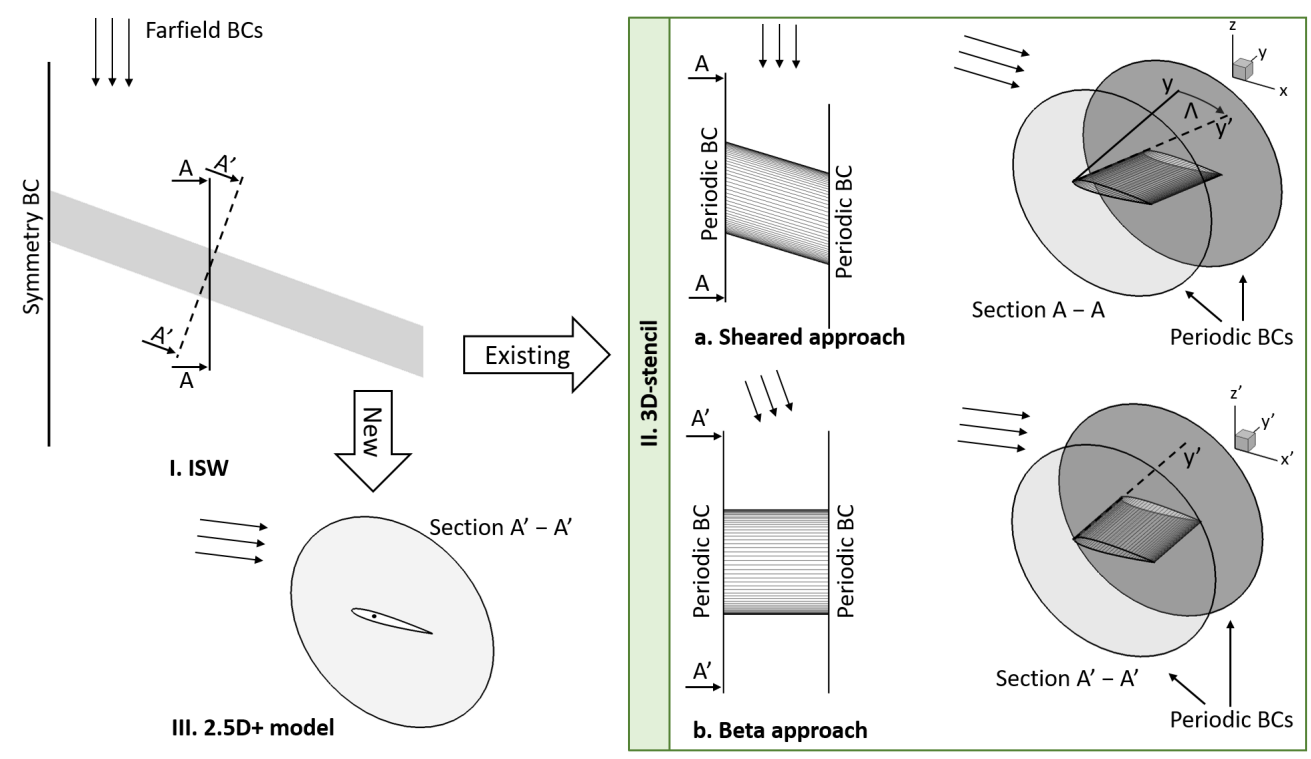

Figure 2: Summary of approaches to model the ISW problem; the "sheared" and "beta" approaches employ a one-cell width 3D stencil; the proposed approach, on the other hand, uses a purely $2 \mathrm{D}$ stencil

equations, reported here in component notation for clarity, become

$$
\begin{gathered}
\frac{\partial \rho}{\partial t}+\frac{\partial\left(\rho u^{\prime}\right)}{\partial x^{\prime}}+\frac{\partial\left(\rho w^{\prime}\right)}{\partial z^{\prime}}=0 \\
\frac{\partial\left(\rho u^{\prime}\right)}{\partial t}+\frac{\partial\left(\rho u^{\prime} u^{\prime}\right)}{\partial x^{\prime}}+\frac{\partial\left(\rho u^{\prime} w^{\prime}\right)}{\partial z^{\prime}}=-\frac{\partial p}{\partial x^{\prime}}+\frac{\partial \tau_{x x}^{\prime}}{\partial x^{\prime}}+\frac{\partial \tau_{x z}^{\prime}}{\partial z^{\prime}} \\
\frac{\partial\left(\rho v^{\prime}\right)}{\partial t}+\frac{\partial\left(\rho u^{\prime} v^{\prime}\right)}{\partial x^{\prime}}+\frac{\partial\left(\rho v^{\prime} w^{\prime}\right)}{\partial z^{\prime}}=\frac{\partial \tau_{y x}^{\prime}}{\partial x^{\prime}}+\frac{\partial \tau_{y z}^{\prime}}{\partial z^{\prime}} \\
\frac{\partial\left(\rho w^{\prime}\right)}{\partial t}+\frac{\partial\left(\rho u^{\prime} w^{\prime}\right)}{\partial x^{\prime}}+\frac{\partial\left(\rho w^{\prime} w^{\prime}\right)}{\partial z^{\prime}}=-\frac{\partial p}{\partial z^{\prime}}+\frac{\partial \tau_{z x}^{\prime}}{\partial x^{\prime}}+\frac{\partial \tau_{z z}^{\prime}}{\partial z^{\prime}} \\
\frac{\partial\left(\rho e_{0}\right)}{\partial t}+\frac{\partial\left(\rho h_{0} u^{\prime}\right)}{\partial x^{\prime}}+\frac{\partial\left(\rho h_{0} w^{\prime}\right)}{\partial z^{\prime}}= \\
\frac{\partial}{\partial x^{\prime}}\left(u^{\prime} \tau_{x x}^{\prime}+v^{\prime} \tau_{x y}^{\prime}+w^{\prime} \tau_{x z}^{\prime}\right)+\frac{\partial}{\partial z^{\prime}}\left(u^{\prime} \tau_{z x}^{\prime}+v^{\prime} \tau_{z y}^{\prime}+w^{\prime} \tau_{z z}^{\prime}\right)+ \\
-\frac{\partial \dot{q}_{x}^{\prime}}{\partial x^{\prime}}-\frac{\partial \dot{q}_{z}^{\prime}}{\partial z^{\prime}}
\end{gathered}
$$

Equations 5a, 5b and (5d) are independent from the cross-flow component, $v^{\prime}$, and are equivalent to the 2D NS equations. Equation (5c) represents a transport equation for the crossflow on the flow field, and can be solved at each iteration once the flow field variables $u^{\prime}$ and $w^{\prime}$ are known. The coupling between cross-flow and the other variables is provided by the energy conservation equation, Eq. (5e). This equation couples the thermal field with the kinematic field, and the effects become significant with compressibility, i.e. Mach numbers above 0.3. It is worth observing that the cross-flow variable, $v^{\prime}$, does not influence only the energy equation 
through the viscous terms, but also modifies the total energy $e_{0}$ and the total enthalpy $h_{0}$ definitions. The numerical solution obtained with this set of equations has the same properties as that obtained using the one-cell width 3D stencil, but instead of leaving the solver to converge to a $y^{\prime}$-independent solution through the periodicity, such information is added in the equations directly.

Note also that this assumption holds true for the solution of the laminar fields. The case of Reynolds-averaged fields will be discussed in Section 3.2. For other modelling techniques, such as large eddy and direct numerical simulations, the three-dimensionality of the flow needs to be solved also in $y^{\prime}$-statistical homogeneity condition, requiring an adequate spanwise resolution.

The system of equations $5 \mathrm{a}-5 \mathrm{e}$ ) can be compactly written in vector form using a modified nabla operator, defined as

$$
\nabla^{\prime}=\left(\frac{\partial}{\partial x^{\prime}}, 0, \frac{\partial}{\partial z^{\prime}}\right)
$$

hence the equations in vector form are

$$
\begin{gathered}
\frac{\partial \rho}{\partial t}+\nabla^{\prime} \cdot\left(\rho \mathbf{V}^{\prime}\right)=0 \\
\frac{\partial\left(\rho \mathbf{V}^{\prime}\right)}{\partial t}+\nabla^{\prime} \cdot\left(\rho \mathbf{V}^{\prime} \otimes \mathbf{V}^{\prime}\right)=-\nabla^{\prime} p+\nabla^{\prime} \cdot \boldsymbol{\Pi}^{\prime} \\
\frac{\partial\left(\rho e_{0}\right)}{\partial t}+\nabla^{\prime} \cdot\left(\rho \mathbf{V}^{\prime} h_{0}\right)=\nabla^{\prime} \cdot\left(\mathbf{V}^{\prime} \cdot \boldsymbol{\Pi}^{\prime}\right)-\nabla^{\prime} \cdot \dot{\mathbf{q}}
\end{gathered}
$$

For aerodynamic laminar flows, the viscous stress tensor can be defined recalling the Newtonian approximation, which assumes a linear, homogeneous and isotropic relation with the deformation tensor which can be written in the body-attached FoR as

$$
\boldsymbol{\Pi}^{\prime}=2 \mu\left(\mathbf{S}^{\prime}-\frac{1}{3} \operatorname{Tr}\left(\mathbf{S}^{\prime}\right) \mathbf{I}\right)
$$

where $\boldsymbol{\Pi}^{\prime}$ is the shear stress tensor, $\mathbf{S}^{\prime}$ indicates the velocity deformation tensor, while the viscosity coefficient is denoted by $\mu$. The operator $\operatorname{Tr}$ is the trace, and $\mathbf{I}$ is identity matrix. Under the ISW model assumption, the velocity deformation tensor is computed as

$$
\mathbf{S}^{\prime}=\frac{1}{2}\left(\nabla^{\prime} \mathbf{V}^{\prime}+\nabla^{\prime} \mathbf{V}^{\prime T}\right)
$$

where

$$
\nabla^{\prime} \mathbf{V}^{\prime}=\left[\begin{array}{ccc}
\frac{\partial u^{\prime}}{\partial x^{\prime}} & 0 & \frac{\partial u^{\prime}}{\partial z^{\prime}} \\
\frac{\partial v^{\prime}}{\partial x^{\prime}} & 0 & \frac{\partial v^{\prime}}{\partial z^{\prime}} \\
\frac{\partial w^{\prime}}{\partial x^{\prime}} & 0 & \frac{\partial w^{\prime}}{\partial z^{\prime}}
\end{array}\right]
$$

For laminar flows, the system of equations is closed by choosing an appropriate thermodynamic model for the fluid and by coupling the $\dot{\mathbf{q}}$ vector with the thermal field, for example, using the Fourier law. Within the ISW approximation, it can be expressed as

$$
\dot{\mathbf{q}}=\frac{\mu}{\operatorname{Pr}} \nabla^{\prime} h
$$

where $h=c_{p} T$ is the enthalpy, $T$ is the temperature, and $\operatorname{Pr}$ is the Prandtl number. 
184

\subsection{RANS Modelling}

Following the steps discussed for the NS equations, one can derive a revised version of the Favre-averaged NS equations for the ISW problem. Such derivation imposes the condition $\partial(\cdot) / \partial y^{\prime}=0$ within the equations similarly to what has been shown in Sec. 3.1. In this case, the additional turbulent stresses can be modelled through the Reynolds stress tensor, and therefore $\mathbf{\Pi}^{\prime}$, evaluated in the body-attached FoR, becomes

$$
\boldsymbol{\Pi}^{\prime}=2 \mu_{e}\left(\mathbf{S}^{\prime}-\frac{1}{3} \operatorname{Tr}\left(\mathbf{S}^{\prime}\right) \mathbf{I}\right)
$$

where $\mu_{e}=\mu+\mu_{t}$ is the sum of the molecular and the turbulent viscosities and $\mathbf{S}^{\prime}$ is defined in Eq. (9). The scalar quantity $\mu_{t}$ is modelled through additional transport equations that can be simplified in the case of an ISW calculation with respect to their 3D versions imposing $\partial(\cdot) / \partial y^{\prime}=$ 0 . We remark that those modifications should be intended as a customization rather than a model variant. In other words, all the physical properties of the original, 3D model are retained. In the current study, the 2.5D+ implementation has been verified with two different turbulence closures, the SA [19] and the $\mathrm{k}-\omega$ [20], and these are briefly discussed below.

The SA model written in the body-attached FoR assumes the following fashion

$$
\begin{aligned}
\frac{\partial \tilde{v}}{\partial t}+\nabla^{\prime} \cdot\left(\mathbf{V}^{\prime} \tilde{v}\right) & =c_{b 1}\left(1-f_{t 2}\right) \tilde{S} \tilde{v}-\left[c_{w 1} f_{w}-\frac{c_{b 1}}{\kappa^{2}} f_{t 2}\right]\left(\frac{\tilde{v}}{d}\right)^{2} \\
& +\frac{1}{\sigma}\left[\nabla^{\prime} \cdot\left((v+\tilde{v}) \nabla^{\prime} \tilde{v}\right)+c_{b 2}\left(\nabla^{\prime} \tilde{v}\right)^{2}\right]
\end{aligned}
$$

and $\mu_{t}=\rho \tilde{v} f_{v 1}$. Here, $v=\mu / \rho, \tilde{S}=\Omega+\tilde{v} /\left(\kappa^{2} d^{2}\right) f_{v 2}$, with

$$
\Omega=\sqrt{2 \mathbf{W}: \mathbf{W}}, \quad \mathbf{W}=\frac{1}{2}\left(\nabla^{\prime} \mathbf{V}^{\prime}-\nabla^{\prime} \mathbf{V}^{\prime T}\right),
$$

which neglects the $y^{\prime}$-derivatives. Note that all the other model coefficients of Ref. [19] are employed.

Similar considerations are valid for the $\mathrm{k}-\omega$ model. The transport equations under the ISW approximation can be written as

$$
\frac{\partial \rho k}{\partial t}+\nabla^{\prime} \cdot\left(\rho \mathbf{V}^{\prime} k\right)=P_{k}-\beta^{*} \rho \omega k+\nabla^{\prime} \cdot\left(\left(\mu+\sigma_{k} \mu_{t}\right) \nabla^{\prime} k\right)
$$

$$
\begin{aligned}
\frac{\partial \rho \omega}{\partial t}+\nabla^{\prime} \cdot\left(\rho \mathbf{V}^{\prime} \omega\right) & =\frac{\gamma}{v_{t}} P_{k}-\beta^{*} \rho \omega^{2}+\nabla^{\prime} \cdot\left(\left(\mu+\sigma_{\omega} \mu_{t}\right) \nabla^{\prime} \omega\right) \\
& +2\left(1-F_{1}\right) \rho \sigma_{\omega 2} \frac{1}{\omega} \nabla^{\prime} k \cdot \nabla^{\prime} \omega
\end{aligned}
$$

and the turbulent viscosity is obtained as $\mu_{t}=\rho k / \omega$. The production term is computed, according to Ref. [20], as $P_{k}=\boldsymbol{\Pi}_{R}^{\prime}: \nabla^{\prime} \mathbf{V}^{\prime}$, which again neglects the $y^{\prime}$-derivatives. Herre $\boldsymbol{\Pi}_{R}^{\prime}$ is the Reynolds stress tensor

$$
\boldsymbol{\Pi}_{R}^{\prime}=2 \mu_{t}\left(\mathbf{S}^{\prime}-\frac{1}{3} \operatorname{Tr}\left(\mathbf{S}^{\prime}\right) \mathbf{I}\right)-\frac{2}{3} \rho k \mathbf{I} .
$$


Note that the shear-stress correction

$$
\mu_{t}=\min \left(\frac{\rho k}{\omega}, \frac{a_{1} \rho k}{\Omega F_{2}}\right)
$$

is computed according to the ISW approximation using $\Omega$ according to Eq. (14). All the other model constants are evaluated according to Ref. [20].

Finally, the heat flux vector $\dot{\mathbf{q}}$ is obtained as

$$
\dot{\mathbf{q}}=\left(\frac{\mu}{\operatorname{Pr}}+\frac{\mu_{t}}{\operatorname{Pr}_{t}}\right) \nabla^{\prime} h
$$

where $\operatorname{Pr}_{t}$ is the turbulent Prandtl number. Additional terms, which are neglected when using the SA model, originate by the mass-weighted average and involve the contribution of the turbulent kinetic energy $k$ transport to the energy equation. They can however be computed again neglecting the $y^{\prime}$-derivative as $\nabla^{\prime} \cdot\left(\sigma_{k} \nabla^{\prime} k\right)$.

\subsection{Boundary Conditions}

The solution of the $2.5 \mathrm{D}+$ governing equations requires appropriate boundary conditions to be defined. For viscous flows, the no-slip boundary condition must be satisfied at the surface by imposing that the three components of the flow velocity are equal to the wall velocity in the body-attached FoR. In case of a stationary wall, $u^{\prime}=v^{\prime}=w^{\prime}=0$, whereas for moving grid problems, the flow velocity at the wall is set equal to the solid surface velocity.

The far-field velocity needs to be imposed in the body-attached FoR. As the body-attached FoR is not aligned with the global FoR, the transformation of the freestream velocity components from the global FoR to the body-attached FoR is required, and is computed using Eq. (2). Standard boundary conditions for the turbulence model quantities can be employed. Furthermore, the Reynolds number in the body-attached FoR needs to be appropriately scaled in order to be consistent with the chord length in the body-attached FoR, using the expression

$$
\operatorname{Re}_{\infty}^{\prime}=\operatorname{Re}_{\infty} \cos (\Lambda)
$$

No scaling of the Mach number is required.

\subsection{Post-processing}

Integrated force coefficients resolved into the body-attached FoR are transformed back to the global FoR using the relation

$$
\left(\begin{array}{l}
C_{x} \\
C_{y} \\
C_{z}
\end{array}\right)=\left[\begin{array}{ccc}
\cos (\Lambda) & \sin (\Lambda) & 0 \\
-\sin (\Lambda) & \cos (\Lambda) & 0 \\
0 & 0 & 1
\end{array}\right]\left(\begin{array}{l}
C_{x}^{\prime} \\
C_{y}^{\prime} \\
C_{z}^{\prime}
\end{array}\right)
$$

which also applies to the moment coefficients. 


\subsection{Numerical Implementation}

The specific numerical implementation of the 2.5D+ approach depends on the type of solver available. Here, two scenarios are considered for exemplification. In the first scenario, one starts with a 2D flow solver. The additional terms related to the cross-flow component, $v^{\prime}$, are introduced into the energy equation and in the turbulence models, where only the source term is affected. The set of equations are solved for the cross-flow in a coupled or segregated manner using the additional transport equation, Eq. (5c). This transport equation can be solved together with the turbulence model in a similar fashion. In the second scenario, featuring a 3D flow solver as starting point, all the transport equations are already implemented. At the pre-processing stage, the $2.5 \mathrm{D}+$ option may be activated reducing the number of element faces employed in the calculations, i.e. only those faces having a normal perpendicular to the direction of periodicity. This yields a significant computational speed-up compared to a solution of the ISW problem on a $3 \mathrm{D}$ stencil.

The numerical implementation of the 2.5D+ method in the DLR-Tau code follows the second approach. This choice reflects a practical situation, with a $3 \mathrm{D}$ solver being available to the authors. Note however that no differences will be observed from the two implementation options discussed above.

\section{Results}

This Section contains a number of test cases to validate the proposed methodology and to assess the expected potential efficiency improvements when compared to an existing state-ofthe-art solver. Section 4.1, in particular, concerns the solution of steady flow problems while Section 4.2 addresses an unsteady flow problem. In all subsequent figures and tables, results obtained from the 2.5D+ method will be labelled as "2.5D+", and those from the existing approach (either "sheared" or "beta") as "3D-stencil". Note that both the $2.5 \mathrm{D}+$ and the $3 \mathrm{D}$ stencil approaches have similar CFL limits, being the scale on the span-wise direction greater than those in the $x^{\prime}-z^{\prime}$ plane. All the tests were performed with the same CFL number, ensuring the same pseudo-time step size within the DLR-Tau framework.

\subsection{Steady-state Results}

This Section presents the application to three test cases. The first test case, discussed in Section 4.1.1, is for a natural laminar flow (NLF) aerofoil in low speed aerodynamics. In the absence of experimental data, the accuracy and computational performance of the 2.5D+ method is compared with the existing state-of-the-art method in solving the ISW problem. Section 4.1.2 introduces the second test case which is the baseline aerofoil of the Onera AFV D variable sweep angle wing. With a number of experimental data available, the comparison is drawn with wind tunnel measurements. Finally, Section 4.1 .3 presents the application to a multi-element wing section extracted from the DLR-F11 high-lift wing-body configuration.

To quantify the computational speed-up of the proposed 2.5D+ method in comparison with the existing state-of-the-art solver, a metric is introduced. The speed-up factor, $\mathcal{S}$, is defined as

$$
\mathcal{S}=\frac{t_{3 \mathrm{D}}}{t_{2.5 \mathrm{D}+}}
$$

For the same convergence level, the term $t_{3 \mathrm{D}}$ indicates the CPU time required by the existing 3D stencil methods (either "beta" or "sheared") to achieve converge. The term $t_{2.5 \mathrm{D}+}$ is the equivalent 
counterpart recording the CPU time required by the 2.5D+ method. By definition, a speed-up factor greater than unity indicates that the $2.5 \mathrm{D}+$ method is computationally more efficient than existing state-of-the-art solvers of the ISW problem while achieving the same accuracy level.

\subsubsection{Natural Laminar Flow Aerofoil}

The NLF aerofoil is based on the NLF(2)-0415 geometry, which is typically used for small commercial aircraft. The freestream boundary conditions are for $M_{\infty}=0.044, \alpha_{\infty}=-2.8 \mathrm{deg}$, and $\operatorname{Re}_{\infty}=1.96 \cdot 10^{6}$. Transition is fixed at $62 \%$ of the chord on the suction side, and fully turbulent flow is assumed on the pressure side. The assessment of the methods is carried out for a sweep angle $\Lambda=45.0 \mathrm{deg}$. The unstructured hybrid grid used in this work consists of 27,077 elements and is shown in Fig. 3 This grid, which was generated using the best industrial practice, was found adequate to guarantee grid independent results.

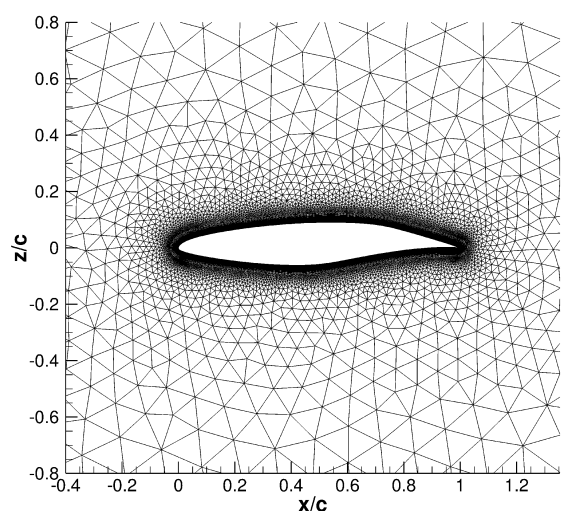

Figure 3: Unstructured hybrid grid of the NLF(2)-0415 aerofoil

To assess the accuracy and computational efficiency, the $2.5 \mathrm{D}+$ method is compared with the 3D stencil approach. The same numerical settings are used in both approaches to ensure a representative comparison. The central scheme with scalar dissipation [21] was used, with second order dissipation coefficient set to 0.5 and inverse fourth order dissipation coefficient set to 64 . The original SA turbulence model was used for the closure of the equations.

First, the accuracy of the methods was investigated. Figure 4 shows the comparison of surface quantities computed with the $2.5 \mathrm{D}+$ and $3 \mathrm{D}$ stencil methods. Identical results are found for the pressure coefficient distribution, shown in Fig. 4(a), and for the components of the skin friction coefficient, in Fig. 4(b). Note that an excellent agreement is also observed near the leading edge, where the gradients are the highest. The comparison validates the $2.5 \mathrm{D}+$ implementation which produces results consistent with the state-of-the-art methods.

Next, the computational efficiency (in terms of CPU-time needed to achieve a target convergence level) is assessed. Figure 5 reports the converge history of the two solvers. The calculations were run until the density residual dropped 13 orders of magnitude (target convergence: $10^{-13}$ ). The convergence of the residual with the number of iterations, shown in Fig. 5(a), reveals a similar general trend. Both solvers achieve the target convergence level in about 12,000 iterations. The computational cost required by the two solvers to achieve full convergence is 


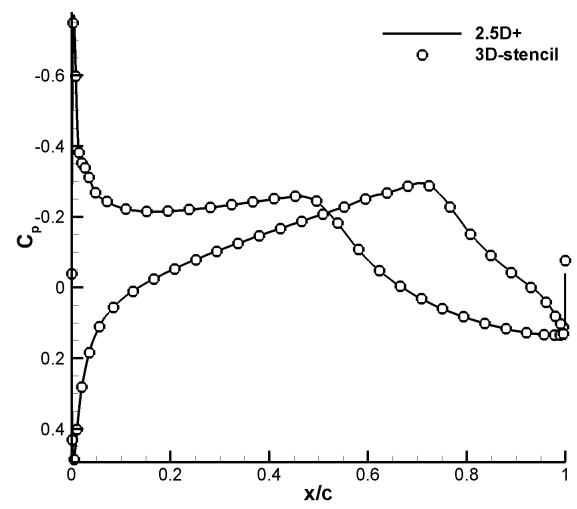

(a) Surface pressure coefficient

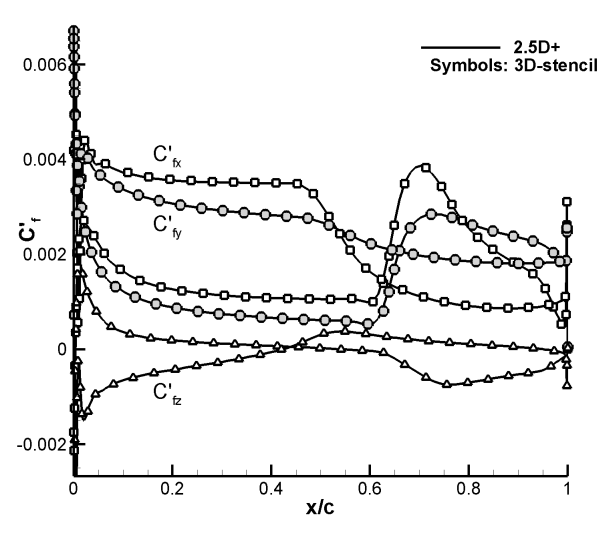

(b) Skin friction components

Figure 4: $\operatorname{NLF}(2)-0415$ aerofoil, surface quantities $\left(M_{\infty}=0.044, \alpha_{\infty}=-2.8 \mathrm{deg}, \operatorname{Re}_{\infty}=1.96 \cdot 10^{6}\right.$, and $\left.\Lambda=45.0 \mathrm{deg}\right)$

compared in Fig. 5(b), where the CPU time of each solver is normalised by the time required by the $2.5 \mathrm{D}+$ strategy to achieve the desired convergence, $t_{0}$. The comparison demonstrates the higher efficiency of the proposed 2.5D+ solver compared to the current state-of-the-art method.

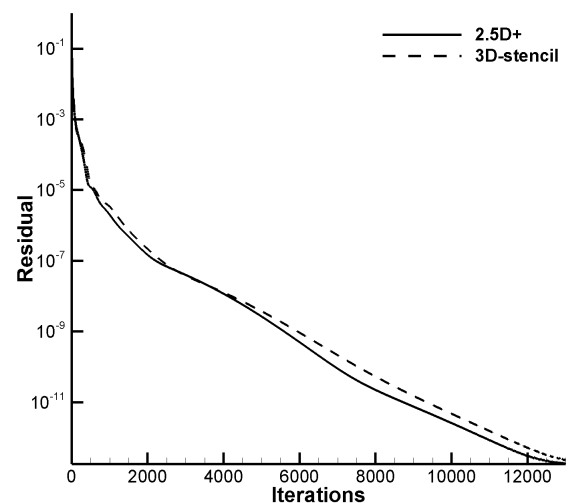

(a) Convergence in iterations

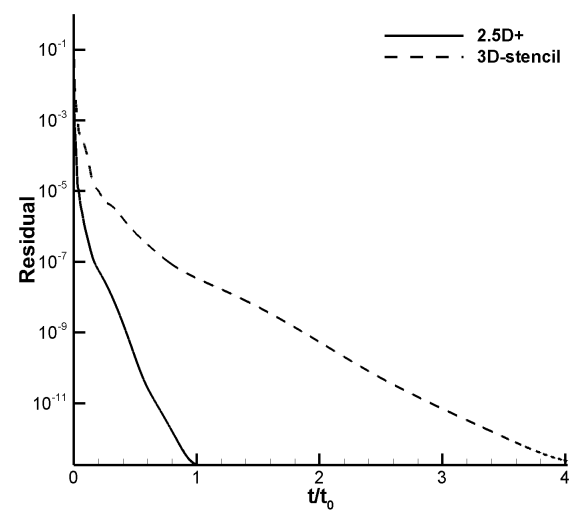

(b) Convergence in normalised time

Figure 5: NLF(2)-0415 aerofoil, convergence analysis $\left(M_{\infty}=0.044, \alpha_{\infty}=-2.8 \mathrm{deg}, \operatorname{Re}_{\infty}=1.96 \cdot 10^{6}\right.$, and $\Lambda=45.0$ deg)

The efficiency of the 2.5D+ implementation is quantified in Table 1 . The speed-up factor, $\mathcal{S}$, and the number of iterations, $n_{i t}$, to achieve three representative residual levels $\left(10^{-9}, 10^{-10}\right.$ and $10^{-13}$ ) are summarised. It was found that the $2.5 \mathrm{D}+$ method is consistently more efficient than the existing method, with a factor larger than 4 . This is equivalent to a reduction of the 
computational time of about $75 \%$. This reflects the decreased complexity of the set of equations solved in the $2.5 \mathrm{D}+$ approach, without impacting on the accuracy despite the different stencils employed.

Table 1: Computational details for the results of the NLF(2)-0415 aerofoil test case
\begin{tabular}{l|ccccccc}
\hline Residual & \multicolumn{3}{c}{$10^{-9}$} & & $10^{-10}$ & \multicolumn{1}{c}{$10^{-13}$} \\
\hline & $\mathcal{S}$ & $n_{i t}$ & $\mathcal{S}$ & $n_{i t}$ & $\mathcal{S}$ & $n_{i t}$ \\
\hline 3D-stencil & - & 5,951 & - & 7,537 & - & 12,262 \\
2.5D+ & 4.36 & 5,596 & 4.43 & 6,934 & 4.23 & 12,900 \\
\hline
\end{tabular}

\subsubsection{OneraD Aerofoil}

The OneraD aerofoil represents the baseline profile for the Onera AFV D variable sweep angle wing as well as the Onera M6 wing. A large body of experimental and numerical work exists, but here the interest is on the Onera AFV D variable sweep angle wing which has been extensively wind tunnel tested [22]. The wing represents a valuable validation test case because it has an aspect ratio (AR) of eight, in the unswept configuration, and a taper ratio of one, satisfying reasonably well the assumptions of the ISW problem without the need for the LLT or VLM.

The numerical settings employed were the same as the previous test case. For the turbulence model, the original SA formulation was used. The flow conditions, for which experimental data are available for a number of angle of attacks, are for $M_{\infty}=0.78$ and $\operatorname{Re}_{\infty}=2.5 \cdot 10^{6}$. The wing configuration was tested for sweep angles of 30 and $50 \mathrm{deg}$. Without other information, the flow was assumed fully turbulent.

Calculations were run on an unstructured hybrid grid containing 93,347 cells, shown in Fig.6. The circular far-field is placed at fifty times the aerofoil chord. The first layer on the wall was placed at $5 \cdot 10^{-6}$ (for a chord of one), ensuring $y^{+}<1$. The spacing distribution was tested to guarantee grid-independent results in terms of lift coefficient for $\alpha_{\infty}=0 \mathrm{deg}$.

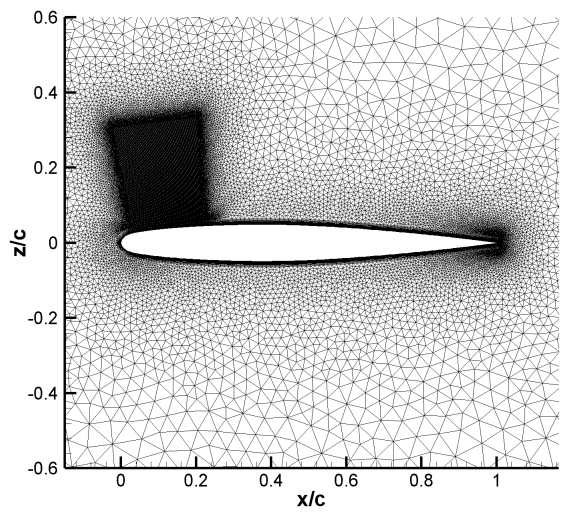

Figure 6: Unstructured grid of the OneraD aerofoil

Experimental data of the pressure coefficient were measured at several sections along the 
span of the Onera AFV D wing. Since the 3D nature of the flow features influences the pressure distribution over the wing span, the comparison between numerical predictions and experimental data was performed for $\alpha_{\infty}=0 \mathrm{deg}$. For an untwisted wing with a symmetric aerofoil, this condition guarantees independence of the flow characteristics from the span-wise location, avoiding wing tip effects. Experimentally, variations of the pressure coefficient on different pressure taps locations were observed, and are attributed to aleatory uncertainties in the model geometry (manufacturing tolerances) and flow conditions (misalignment, blockage, etc.). Another concurrent factor is the wing sweep angle which promotes the occurrence of cross-flow effects that are affected by the conditions at the two boundaries (wall on one side and free wing tip on the other).

Figure 7 shows the pressure coefficient distribution at a span-wise section, $y / b=0.6$, where the flow is considered fully developed. Calculations were run to machine accuracy. Pressure tap measurements are reported in symbols, and lines indicate $2.5 \mathrm{D}+$ results. A good agreement is observed for the three sweep angles tested $(\Lambda=0,30,50 \mathrm{deg})$. The $2.5 \mathrm{D}+$ predictions capture relevant physical effects, such as the pressure recovery at the cove region and the decreasing intensity of the shock at the leading edge for increasing sweep angles.

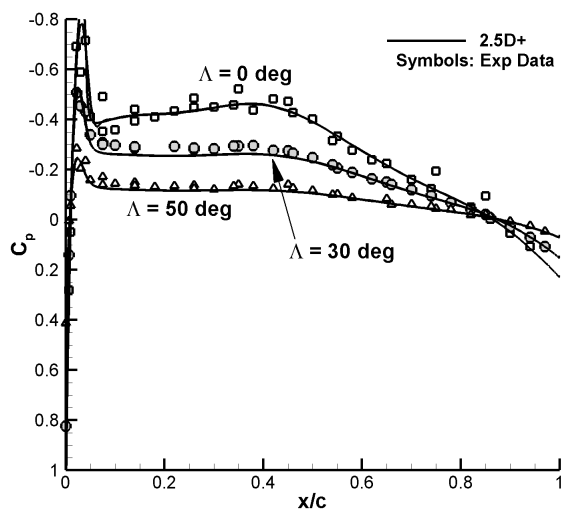

Figure 7: Onera AFV D wing, pressure coefficient distribution at span-wise station $y / b=0.6\left(M_{\infty}=0.78, \alpha_{\infty}=0.0\right.$ $\mathrm{deg}$, and $\operatorname{Re}_{\infty}=2.5 \cdot 10^{6}$ ); experimental data from Ref. [22]

The computational performance of the 2.5D+ method is assessed against the 3D stencil approach. To ensure a sufficient generality of the assessment, the comparison is performed at three angles of attack ( $\alpha_{\infty}=0,2.59$, and $4.33 \mathrm{deg}$, corresponding to $\alpha_{\infty}^{\prime}=0,3$ and $5 \mathrm{deg}$ ). For the $\Lambda=30 \mathrm{deg}$ configuration, the predictions of the flow solution are reported in Fig. 8 . For the pressure coefficient, the virtually perfect match between the two approaches validates again the implementation. Despite the fact that the stencil of the $2.5 \mathrm{D}+$ method is purely $2 \mathrm{D}$, the position and intensity of the shock are captured in the same way as the one-cell width 3D stencil approach.

Figure 9 shows the flow-field solution for the $\Lambda=30 \mathrm{deg}$ wing at a free-stream incidence $\alpha_{\infty}=4.33 \mathrm{deg}$. The upper plots illustrate the pressure coefficient and cross-flow velocity component computed with the $2.5 \mathrm{D}+$ approach. The lower plots are for the difference in the flow-field solutions between the 2.5D+ and 3D-stencil approaches. These differences, which are limited to the shock region, occur across one grid cell. The reason for this is attributed to the slightly 


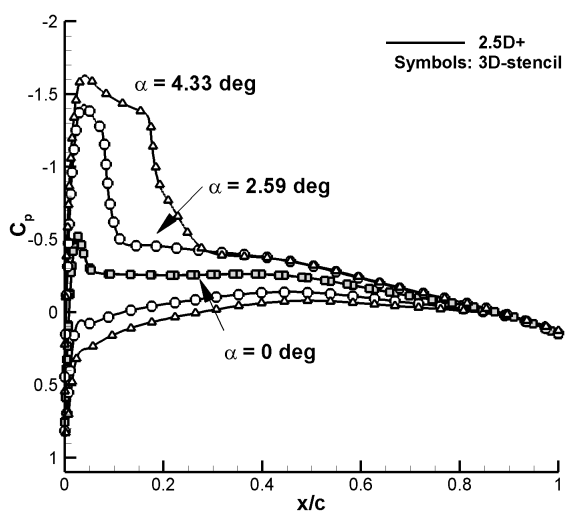

Figure 8: Onera D aerofoil, pressure coefficient distribution $\left(M_{\infty}=0.78, \operatorname{Re}_{\infty}=2.5 \cdot 10^{6}\right.$, and $\left.\Lambda=30.0 \mathrm{deg}\right)$

different dissipative behaviour of the spatial discretisations employed in the two approaches. The flow field indicates that the intensity of the cross-flow decreases behind the shock position due to flow separation. The qualitative match corroborates previous considerations on the accuracy of the $2.5 \mathrm{D}+$ method. Similar observations were found for the configuration with $\Lambda=50 \mathrm{deg}$. With decreasing intensity of the leading edge shock, the flow remains attached. Comparisons are not reported herein for brevity.
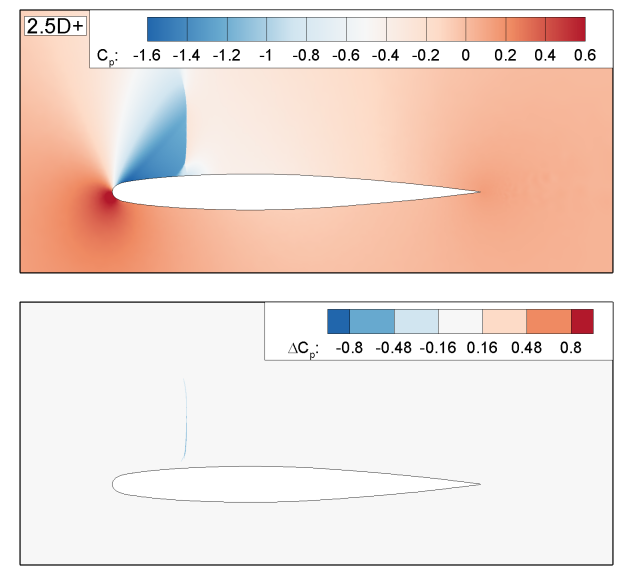

(a) Pressure coefficient
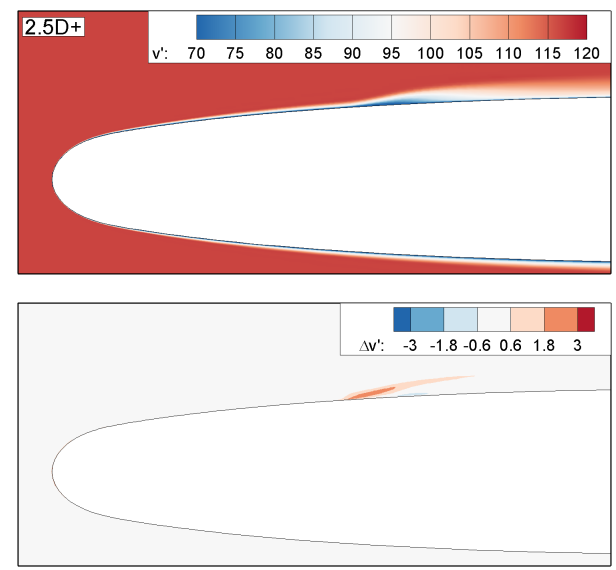

(b) Cross-flow velocity component, $v^{\prime}$

Figure 9: Onera D aerofoil, flow field solutions (top) and error (bottom) $\left(M_{\infty}=0.78, \alpha_{\infty}=4.33 \mathrm{deg}, \operatorname{Re}_{\infty}=2.5 \cdot 10^{6}\right.$, and $\Lambda=30 \mathrm{deg}$ )

Figure 10 summarises the convergence history of the density residual plotted versus nondimensional CPU time for the three angles of attack $\left(\alpha_{\infty}^{\prime}=0,3\right.$ and $\left.5 \mathrm{deg}\right)$ and two configurations $\Lambda=30$ and $50 \mathrm{deg}$ ). An observation is that the angle of attack has a negligible influence on the 
convergence rate of both solvers. It is also observed that the higher computational efficiency of the $2.5 \mathrm{D}+$ solver remains nearly constant with the sweep angle.

A quantitative analysis of the computational efficiency is reported in Table 2 for the $30 \mathrm{deg}$ sweep angle case, and in Table 3 for the $50 \mathrm{deg}$ test case. Note that the speed-up is assessed for the target residual of $10^{-8}$. For all cases tested, the $2.5 \mathrm{D}+$ method retains the higher computational efficiency already observed for the NLF(2)-0415 aerofoil. For this transonic fully turbulent problem, in particular, the CPU-time using the $2.5 \mathrm{D}+$ solver is reduced by more than $75 \%$ compared with the cost required by the existing 3D stencil approach. As the number of iterations to achieve convergence are comparable, the cost of each iteration of the $2.5 \mathrm{D}+$ solver is on average more than $75 \%$ faster than the that of the 3D stencil method.

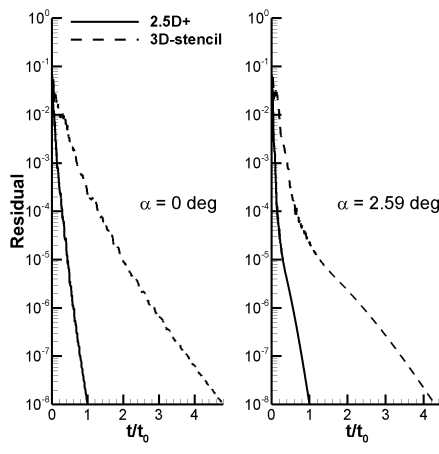

(a) $\Lambda=30 \mathrm{deg}$
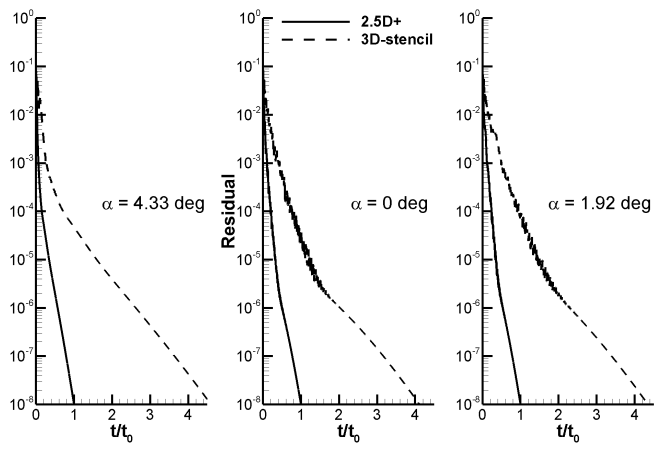

(b) $\Lambda=50 \mathrm{deg}$

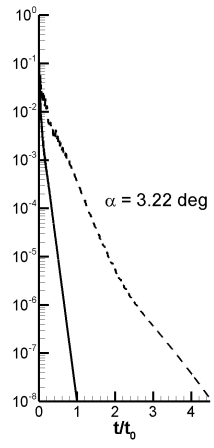

Figure 10: Onera D aerofoil, convergence analysis $\left(M_{\infty}=0.78\right.$ and $\left.\operatorname{Re}_{\infty}=2.5 \cdot 10^{6}\right)$

\begin{tabular}{l|cccccc}
\hline \hline$\alpha_{\infty}[\mathrm{deg}]$ & \multicolumn{2}{|c}{0} & \multicolumn{2}{c}{2.59} & \multicolumn{2}{c}{4.33} \\
\hline & $\mathcal{S}$ & $n_{i t}$ & $\mathcal{S}$ & $n_{i t}$ & $\mathcal{S}$ & $n_{i t}$ \\
\hline 3D-stencil & - & 44,057 & - & 39,654 & - & 43,633 \\
$2.5 \mathrm{D}+$ & 4.79 & 45,460 & 4.30 & 40,012 & 4.60 & 43,902 \\
\hline \hline
\end{tabular}

Table 3: Computational efficiency analysis for the Onera $\mathrm{D}$ aerofoil $(\Lambda=50 \mathrm{deg})$

\begin{tabular}{l|cccccc}
\hline \hline$\alpha_{\infty}$ [deg] & \multicolumn{2}{|c}{0} & \multicolumn{2}{c}{1.92} & \multicolumn{2}{c}{3.22} \\
\hline & $\mathcal{S}$ & $n_{i t}$ & $\mathcal{S}$ & $n_{i t}$ & $\mathcal{S}$ & $n_{i t}$ \\
\hline 3D-stencil & - & 38,120 & - & 40,000 & - & 41,350 \\
$2.5 \mathrm{D}+$ & 4.11 & 38,695 & 4.35 & 40,443 & 4.55 & 44,522 \\
\hline \hline
\end{tabular}

\subsubsection{Multi-element Aerofoil}

A representative multi-element aerofoil section was extracted from the DLR-F11 configuration which was used in the $2^{\text {nd }}$ AIAA High Lift Prediction Workshop. In particular, the aerofoil 
section is located at a non-dimensional span-wise station $y / b=0.964$. The unstructured mesh which consists of about 43 thousand triangular elements is shown in Fig. 11 .

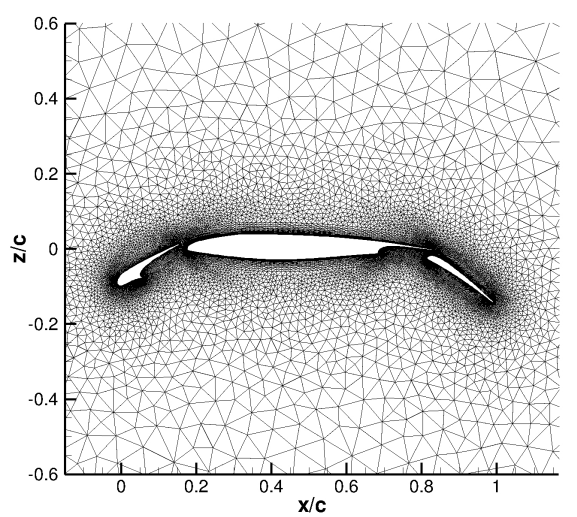

Figure 11: Unstructured grid of the multi-element aerofoil (section at $y / b=0.964$ of the DLR-F11 configuration)

The flow conditions are for $\operatorname{Re}_{\infty}=25 \cdot 10^{6}, M_{\infty}=0.22$. The local wing sweep angle is $\Lambda=30 \mathrm{deg}$, therefore $\alpha_{\infty}^{\prime}=24.5 \mathrm{deg}$. Flow separation is a dominant feature and, to avoid introducing the dependence on computational parameters such as the location of turbulence transition, all cases were run fully turbulent. The SA and $\mathrm{k}-\omega$ models were used.

Figure 12 compares the pressure coefficient distribution obtained using the 2.5D+ and 3D stencil methods for the two turbulence models. The SA model predicts attached flow. On the opposite, the $\mathrm{k}-\omega$ model predicts the flow to be fully separated over the main element. Despite the flow solution is highly dependent on the turbulence model used, as it may be expected in this flow regime, the main point here is the equivalence of the predictions between the two ISW model implementations. A speed-up consistent with the previous test cases was also recorded.

\subsection{Forced Sinusoidal Motion}

The last test case concerns transonic flow predictions for a forced sinusoidal motion. The test case is a prototype problem, being the forced motion representative of (undamped) structural vibrations or the kinematics imposed traditionally to extract aerodynamic derivatives. The flow conditions are related to AGARD CT5 $\left(M_{\infty}=0.755\right.$ and $\left.\operatorname{Re}_{\infty}=5.5 \cdot 10^{6}\right)$ and the forced sinusoidal motion

$$
\alpha_{\infty}(t)=\alpha_{0}+\alpha_{A} \sin (2 k \tau)
$$

is characterised by a reduced frequency $k=0.0814$, mean angle of attack $\alpha_{0}=0.016 \mathrm{deg}$ and amplitude $\alpha_{A}=2.51 \mathrm{deg}[23]$. The usual convention is to denote the non-dimensional time by $\tau$. The flow field presents the formation of a strong and highly dynamic shock wave experiencing Tijdeman and Seebass's [24] type-B shock motion. The steady solution includes a virtually symmetric shock wave which periodically appears and disappears on the upper and lower surfaces as consequence of the harmonic motion.

Two sets of geometries (and grids) were used, see Fig. 13 The first set is employed to solve the 2.5D+ unsteady RANS (URANS) equations. The grid consists of about 15.3 thousand 


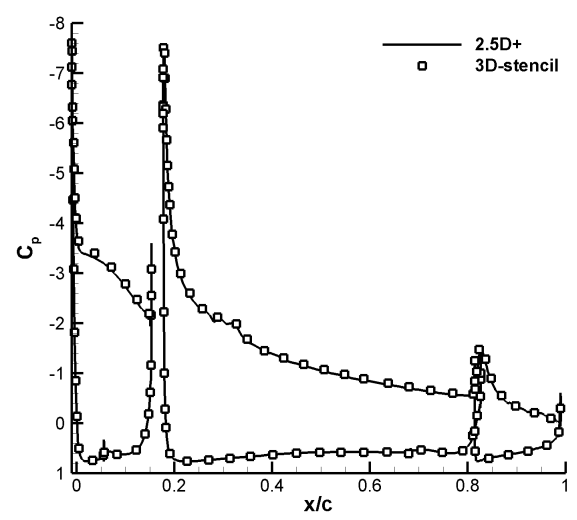

(a) SA turbulence model

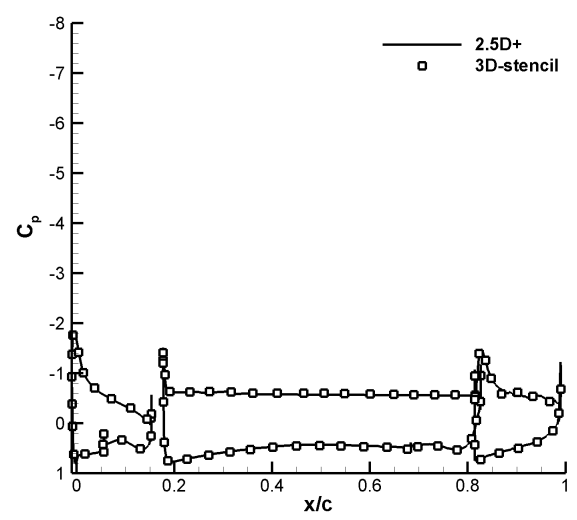

(b) $\mathrm{k}-\omega$ turbulence model

Figure 12: Multi-element aerofoil, surface pressure coefficient $\left(\alpha_{\infty}=21.53 \mathrm{deg}, \operatorname{Re}_{\infty}=25 \cdot 10^{6}, M_{\infty}=0.22, \Lambda=30\right.$ deg)

mesh elements with wall refinement. The first layer on the wall was placed at $5 \cdot 10^{-6}$ (for a chord of one), ensuring $y^{+}<1$. The second set is representative of a 3D half wing model of aspect ratio (AR) 20, see Fig. 13(b) Three sets of 3D wings were generated, for 0, 10, and $20 \mathrm{deg}$ sweep angles. The 3D wing was built by stacking the $2 \mathrm{D}$ unstructured grid in the span-wise direction, from the symmetry plane on one side to the lateral far-field boundary. In the span-wise direction, 128 points were used, featuring about 2 million mesh elements, and a symmetry boundary condition was used to halve the computational cost. For all test cases, the SA turbulence model was employed. An implicit dual-time stepping scheme was used, with a target residual drop of three orders at each physical time step. One hundred time steps per cycle with 600 pseudo iterations were employed, and four cycles were simulated.

The time response of the normal force coefficient for the $2.5 \mathrm{D}+$ solution is shown in Fig. 14 with experimental data. The curve labelled "3D (wing)" is the (sectional) normal force coefficient resulting from the integration of the surface pressure coefficient at the $50 \%$ span-wise section of the $3 \mathrm{D}$ wing. This section is highlighted in red in Fig. 13 . In all cases, the pitch axis is at the quarter chord. Numerical results are in good agreement for this first test case which features zero sweep angle $(\Lambda=0 \mathrm{deg})$. The slightly smaller hysteresis of the $3 \mathrm{D}$ wing reflects $3 \mathrm{D}$ timedependent flow effects, which propagate along the wing span, due to the symmetry boundary condition, on one side, and the free end at the wing tip. Deviations from experimental data have been discussed previously in the open literature [7].

Having performed a validation for the no sweep case against experimental data, we proceed to investigate the effects of the sweep angle $(\Lambda=10$ and $20 \mathrm{deg})$ on the unsteady flow. For the $3 \mathrm{D}$ wing, the axis of rotation is fixed at the quarter of the root chord, as shown in Fig. 15. This figure also illustrates the procedure to impose the correct kinematics for the $2.5 \mathrm{D}+$ method. The aerofoil section is extracted at $50 \%$ of the wing span where the flow is assumed fully developed in the span-wise direction. Due to the offset from the sweep angle at this location, the axis of rotation lies upstream of the aerofoil quarter chord. Furthermore, for $2.5 \mathrm{D}+$ analysis, the forced 


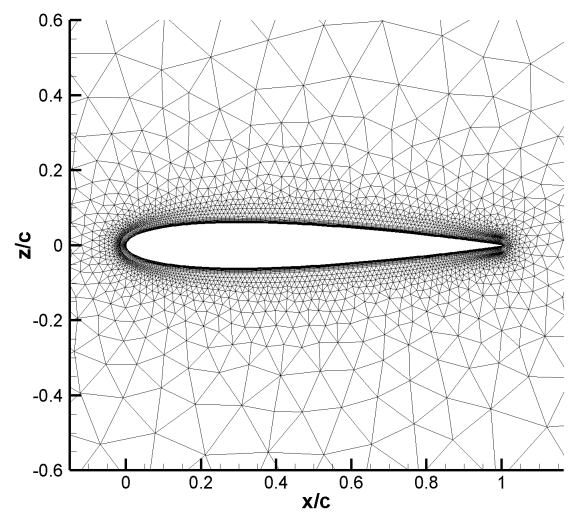

(a) Unstructured 2D grid

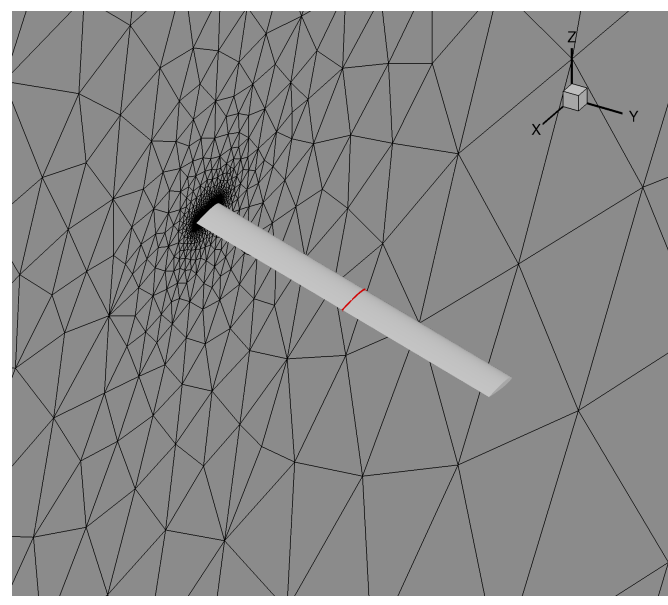

(b) Aspect ratio 20 wing with a 20 deg sweep angle

Figure 13: Grids for the forced sinusoidal motion test case; the cross section is for the NACA 0012 aerofoil

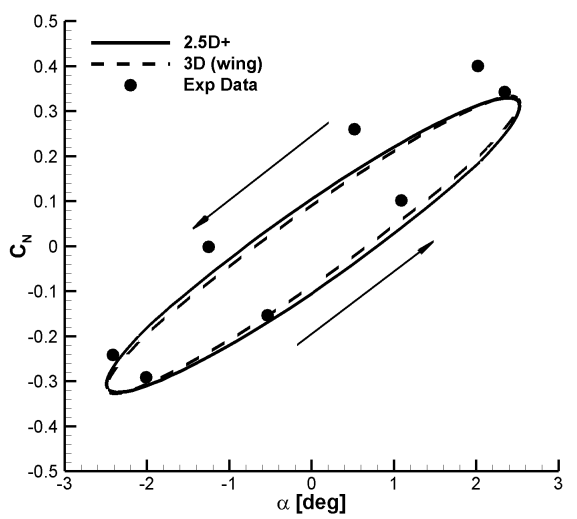

Figure 14: Lift coefficient dependence on forced sinusoidal motion, $\Lambda=0 \mathrm{deg}\left(M_{\infty}=0.755, \operatorname{Re}_{\infty}=5.5 \cdot 10^{6}\right.$, $k=0.0814, \alpha_{0}=0.016 \mathrm{deg}, \alpha_{A}=2.51 \mathrm{deg}$ ); experimental data from Ref. [23] 
I. ISW to body-attached FoR using Eq. (3).

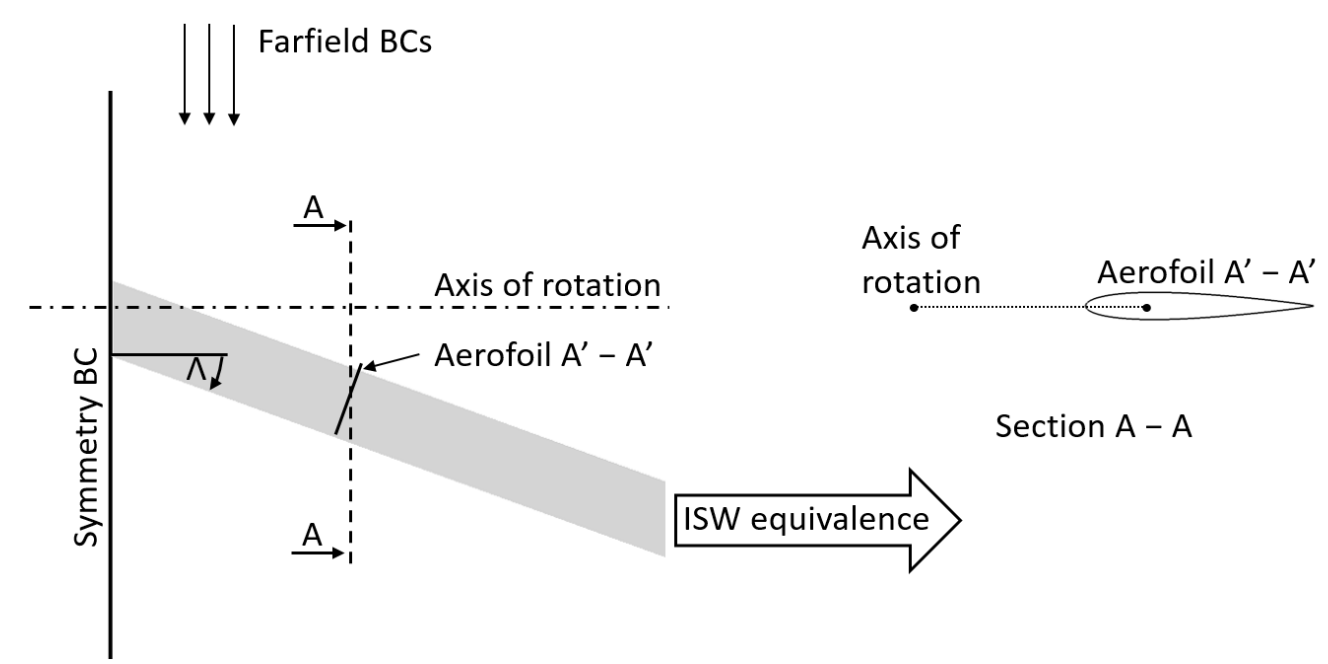

sinusoidal motion given in Eq. 23), which is written in the global FoR, needs to be transformed

II. 2.5D+ model

Figure 15: Kinematics of the forced sinusoidal motion around the axis of rotation

Figure 16 shows the impact of the sweep angle on the time response of the normal force coefficient. The $2.5 \mathrm{D}+$ solutions, computed around the 2D stencil grid, are in remarkably good agreement with the solutions computed around the complete 3D wing. The hysteresis of the loops (aerodynamic damping) decreases for increasing sweep angles, and disappears completely for the $\Lambda=20 \mathrm{deg}$ wing. The reason for this is that, for increasing sweep angle, the axis of rotation moves further upstream of the aerofoil quarter chord. This, in turn, modifies the forced oscillatory motion perceived by the aerofoil section, from a purely pitch oscillation to an oscillatory motion with a dominant plunge component.

The time response to a forced sinusoidal motion is conveniently analysed in the frequency domain employing the Fourier transform. The finite Fourier transform of a continuous scalar function $g(t)$ on a finite time interval $t \in[0, T]$ is defined as

$$
\mathcal{F}[g(t)] \equiv \tilde{g}(i \omega)=\int_{0}^{T} g(t) e^{-i 2 \pi f t} d t
$$

where $i$ is the imaginary unit, and $f$ the dimensional frequency $(2 \pi f=k V / b)$. The system response, quantified by the amplitude ratio and phase lag with respect to the input, can be determined by the transfer function between the input and the output. For the surface pressure coefficient $C_{p}(x, t)$, this is

$$
G(x, \omega)=\frac{\mathcal{F}\left[C_{p}(x, t)\right]}{\mathcal{F}[\alpha(t)]}=R(x, \omega) e^{i \phi(x, \omega)}
$$

where $R(x, \omega)$ and $\phi(x, \omega)$ are the amplitude ratio and phase lag, respectively, and are defined 


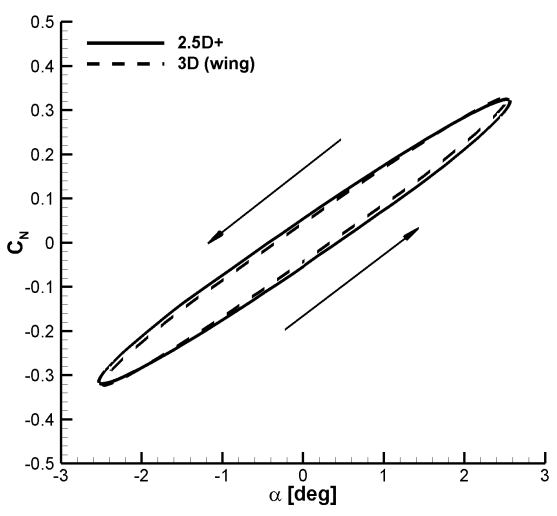

(a) $\Lambda=10 \mathrm{deg}$

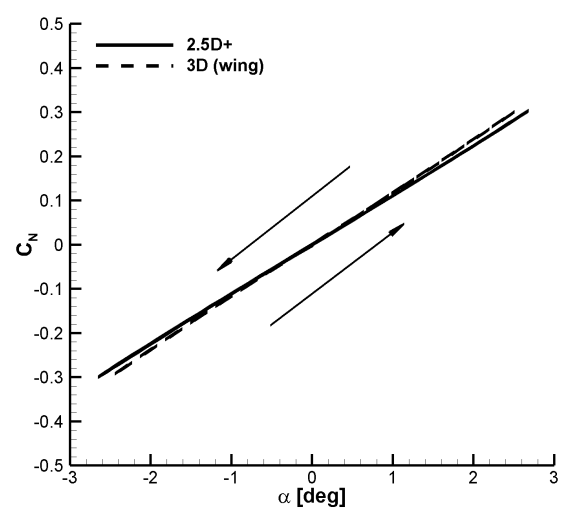

(b) $\Lambda=20 \mathrm{deg}$

Figure 16: Lift coefficient dependence on forced sinusoidal motion for two sweep angles $\left(M_{\infty}=0.755, \operatorname{Re}_{\infty}=5.5 \cdot 10^{6}\right.$, $\left.k=0.0814, \alpha_{0}=0.016 \mathrm{deg}, \alpha_{A}=2.51 \mathrm{deg}\right)$

as

$$
R(x, \omega)=\frac{\left\|\tilde{C}_{p}(x, i \omega)\right\|}{\|\tilde{\alpha}(i \omega)\|}
$$

$$
\phi(x, \omega)=\angle \tilde{C}_{p}(x, i \omega)-\angle \tilde{\alpha}(i \omega)
$$

The transfer function calculations were carried out using a standalone Matlab-based toolbox that has proved accurate in previous studies [25]. This toolbox is freely available from the University of Southampton repository at http://dx.doi.org/10.5258/SOTON/D0272.

Figure 17 quantifies the variation of the transfer function at the oscillatory frequency of interest, $k=0.0814$, for several values of the wing sweep angle. The amplitude ratio of the transfer function at the lower and upper surfaces of the aerofoil are coincident. This is not unexpected because the mean angle of attack is small and the aerofoil section is symmetric. During the harmonic motion, the shock moves upstream and downstream with respect to the average flow field [7], which depends in turn on the wing sweep angle. For the phase angle, the two distinct curves for a given sweep angle reflect the different behaviour on the lower and upper surfaces of the aerofoil. In particular, as the angle of attack increases, the pressure coefficient on the upper side decreases, and that on the lower side increases. In a frequency domain analysis, this observation translates into a phase angle of approximately $\pm 180 \mathrm{deg}$ for the upper aerofoil surface, and a phase angle of approximately $0 \mathrm{deg}$ for the lower surface. Overall, the 2.5D+ method provides a physically consistent solution, with a comparable information content to that obtained from a 3D solution but at a fraction of the computational time. Specifically, 146 CPU hours per oscillatory cycle were needed for the calculation around the 3D wing. The CPU time per cycle was reduced to 30 minutes when employing the $2.5 \mathrm{D}+$ solver. 


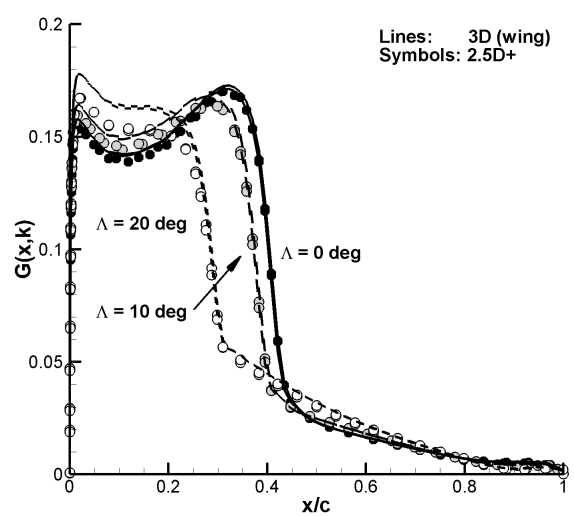

(a) Amplitude ratio

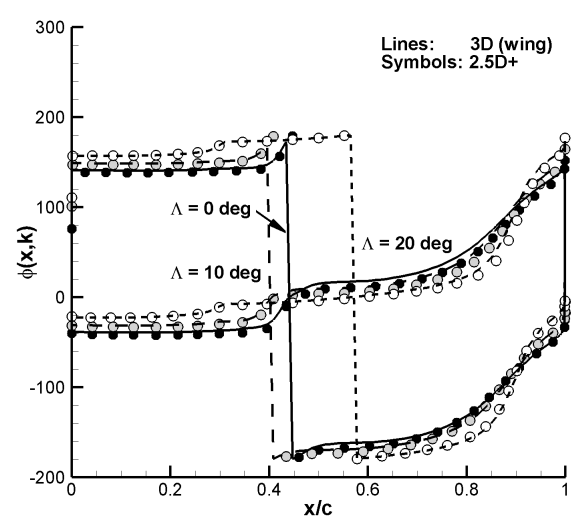

(b) Phase angle

Figure 17: Transfer function at the fundamental harmonic $(k=0.0814)$ between the angle of attack and the surface pressure coefficient $\left(M_{\infty}=0.755, \operatorname{Re}_{\infty}=5.5 \cdot 10^{6}, k=0.0814, \alpha_{0}=0.016 \mathrm{deg}, \alpha_{A}=2.51 \mathrm{deg}\right)$

\section{Conclusions}

An efficient implementation for the solution of the infinite-swept wing problem was discussed. The proposed method was thoroughly validated against experimental data, when available, and numerical data obtained from existing methods. A set of configurations for singleelement and multi-element aerofoils were chosen, in steady and unsteady flow problems. The flow regimes included transitional and fully turbulent conditions.

Two conclusions may be formulated following the extensive validation campaign. The first is that the proposed method retains the same accuracy of existing state-of-art methods, capturing well-known physical phenomena that characterize the flow field around swept wings. The second conclusion is for the high efficiency of the proposed method. The solution of the NavierStokes equations for the infinite-swept wing problem is carried out on a two-dimensional stencil, as opposed to existing methods employing a three-dimensional stencil. The outgrowths of this operation is a computational cost reduction by at least $75 \%$ with a similar convergence of the flow solver. Further application of the proposed method to a geometry of industrial relevance has shown consistent speed-up values larger than that reported herein (up to 97\%).

It is worth observing that the proposed method was implemented in the DLR-Tau flow solver, an industrial grade package used by a number of European aerospace industries. The deployment of the method within an industrial environment is seamless as no changes to current procedures, methods and tools are needed. This highlights the relevance of the present work within an industrial context. Current work concerns the use of the method to correct, via nonlinear sectional data, a three-dimensional linear aerodynamic method, and the coupling with a structural solver for (static and dynamic) aeroelastic analysis. These studies will be reported in a separate publication. 


\section{Acknowledgements}

Da Ronch gratefully acknowledges the financial support from the Royal Academy of Engineering (grant numbers: ISS1415\7\44 and DVF1516|3\16) and the Engineering and Physical Sciences Research Council (grant number: EP/P006795/1). Mr M. Cross and Mr T. Engelbrecht of Airbus Operation Limited are acknowledged for their assistance and comments on industrial practice.

Data supporting this study are openly available from the University of Southampton repository at http://dx.doi.org/10.5258/SOTON/D0272.

\section{References}

[1] A. Rizzi, Modeling and simulating aircraft stability and control - The SimSAC project, Progress in Aerospace Sciences 47 (8) (2011) 573-588, doi 10.1016/j.paerosci.2011.08.004

[2] P. Piperni, A. DeBlois, R. Henderson, Development of a multilevel multidisciplinary-optimization capability for an industrial environment, AIAA journal 51 (10) (2013) 2335-2352, doi $10.2514 / 1 . J 052180$

[3] M. Butchers, Uncertainty quantification \& management in high value manufacturing special interest group, Annual Report, The Knowledge Transfer Network, 2015.

[4] European aviation safety agency, certification specifications for large aeroplanes CS-25. Volume Subpart C - Structure, https://www.easa.europa.eu/ 2008.

[5] A. Da Ronch, M. Panzeri, M. A. Abd Bari, R. d'Ippolito, M. Franciolini, Adaptive design of experiments for efficient and accurate estimation of aerodynamic loads, Aircraft Engineering and Aerospace Technology 89 (4), doi 10.1108 /AEAT-10-2016-0173

[6] A. Da Ronch, M. Ghoreyshi, K. J. Badcock, On the generation of flight dynamics aerodynamic tables by computational fluid dynamics, Progress in Aerospace Sciences 47 (8) (2011) 597-620, doi 10.1016/j.paerosci.2011.09.001

[7] A. Da Ronch, A. J. McCracken, K. J. Badcock, M. Widhalm, M. S. Campobasso, Linear frequency domain and harmonic balance predictions of dynamic derivatives, Journal of Aircraft 50 (3) (2013) 694-707, doi $10.2514 / 1 . C 031674$

[8] S. Timme, K. J. Badcock, A. Da Ronch, Gust load analysis using computational fluid dynamics derived reduced order models, Journal of Fluids and Structures 71 (2017) 116-125, doi 10.1016/j.jfluidstructs.2017.03.004

[9] D. Reckzeh, Aerodynamic design of the high-lift-wing for a megaliner aircraft, Aerospace Science and Technology 7 (2) (2003) 107-119, doi 10.1016/S1270-9638(02)00002-0

[10] G. Trapani, A. M. Savill, T. Kipouros, C. Agostinelli, A. Rampurawala, A rapid approach to the aerodynamic design of a flexible high-lift wing, in: AIAA Aviation, Atlanta, GA, doi:10.2514/6.2014-2011 2014.

[11] A. Elham, M. J. L. van Tooren, Coupled adjoint aerostructural wing optimization using quasi-three-dimensional aerodynamic analysis, Structural and Multidisciplinary Optimization 54 (4) (2016) 889-906, doi 10.1007/s00158016-1447-9

[12] M. Drela, M. B. Giles, Viscous-inviscid analysis of transonic and low Reynolds number airfoils, AIAA journal 25 (10) (1987) 1347-1355, doi 10.2514/3.9789

[13] C. P. Van Dam, The aerodynamic design of multi-element high-lift systems for transport airplanes, Progress in Aerospace Sciences 38 (2) (2002) 101-144, doi 10.1016/S0376-0421(02)00002-7

[14] J. R. Dagenhart, W. S. Saric, Crossflow stability and transition experiments in swept-wing flow, NASA/TP-1999209344, 1999.

[15] S. Bourgault-Côté, S. Ghasemi, A. Mosahebi, É. Laurendeau, Extension of a Two-Dimensional Navier-Stokes Solver for Infinite Swept Flow, AIAA Journal 55 (2) (2017) 1-6, doi 10.2514/1.J055139

[16] D. Schwamborn, T. Gerhold, R. Heinrich, The DLR TAU-code: recent applications in research and industry, in: Proceedings of the European Conference on Computational Fluid Dynamics (ECCOMAS), 2006.

[17] A. Jameson, W. Schmidt, E. Turkel, Numerical solutions of the Euler equations by finite volume methods using Runge-Kutta time-stepping schemes, in: 14th Fluid and Plasma Dynamics Conference, Fluid Dynamics and Colocated Conferences, Palo Alto, CA, doi 10.2514/6.1981-1259. 1981.

[18] J. Drofelnik, A. Da Ronch, 2.5D+ User Guide, DLR-Tau Documentation, 2017.

[19] P. R. Spalart, S. R. Allmaras, A one-equation turbulent model for aerodynamic flows, La Recherche Aérospatiale 1 (1994) 5-21.

[20] F. R. Menter, Two-equation eddy-viscosity turbulence models for engineering applications, AIAA Journal 32 (1994) 1598-1605, doi $10.2514 / 3.12149$ 
[21] D. J. Mavriplis, A. Jameson, Multigrid solution of the Navier-Stokes equations on triangular meshes, AIAA Journal 28 (8) (1990) 1415-1425, doi 10.2514/3.25233

[22] F. Manie, J. Raynal, Transonic measurements on the ONERA AFV D variable sweep wing in the ONERA S2 MA wind tunnel, in: AGARD AR-138, 1979.

[23] R. H. Landon, NACA 0012. Oscillating and transient pitching, Compendium of Unsteady Aerodynamic Measurements, Data Set 3, AGARD-R-702, 1982.

[24] H. Tijdeman, R. Seebass, Transonic flow past oscillating airfoils, Annual Review of Fluid Mechanics 12 (1980) $181-222$.

25] A. Da Ronch, D. Vallespin, M. Ghoreyshi, K. J. Badcock, Evaluation of Dynamic Derivatives Using Computational Fluid Dynamics, AIAA Journal 50 (2) (2012) 470-484, doi $10.2514 / 1 . J 051304$ 